\title{
Quantification of normal head morphometry of stallion spermatozoa
}

\author{
C. G. Gravance ${ }^{\text {I }}$, I. K. M. Liu' ${ }^{2}$, R. O. Davis ${ }^{3}$, J. P. Hughes ${ }^{2}$ and \\ P. J. Casey $^{1,2}$ \\ ${ }^{1}$ Research Centre in Reproductive Medicine, The University of Auckland, Auckland, New Zealand; \\ ${ }^{2}$ School of Veterinary Medicine, Population Health and Reproduction, University of California, Davis CA, \\ USA; and ${ }^{3}$ Reproductive Medicine, Rockford Memorial Hospital, University of Illinois, Rockford IL, USA
}

\begin{abstract}
The heads of stallion spermatozoa were analysed by computer automated sperm head morphometry and the morphometric values of the major subpopulations of sperm heads were assessed. The criteria for normal dimensions of stallion sperm heads are proposed based on the analysis of these measurements. Semen samples were collected from 10 fertile and 10 subfertile stallions, processed by a standard method, smeared onto microscope slides and stained using haematoxylin. At least 200 properly digitized sperm heads were analysed from each stallion. The measurements for length, width, area, perimeter and width/length were recorded for each stallion. All sperm head measurements were placed in a statistical database and multivariate cluster analysis performed. Mean measurements for all parameters of the major clusters of fertile and subfertile stallions were compared by analysis of variance. The ranges of the values of the major clusters of fertile stallions were applied to all stallions to determine the percentage of normal sperm heads for each stallion. The mean values for length, width, area and perimeter in the major cluster of sperm head dimensions of fertile stallions were significantly different from those of the subfertile stallions $(P<0.001)$. The range of values of the major cluster of fertile stallions was length $=4.9-5.7 \mu \mathrm{m}$, width $=2.5-3.0 \mu \mathrm{m}$, width/length $=0.45-0.59, \quad$ area $=10.3-12.1 \mu \mathrm{m}^{2}$, and perimeter $=$ $12.9-14.2 \mu \mathrm{m}$. On the basis of these values, a significantly $(P<0.001)$ higher percentage of normal sperm heads were found in the fertile group than in the subfertile group of stallions $(52 \%$ versus $19 \%)$.
\end{abstract}

\section{Introduction}

The clinical detection of subfertility is extremely important in human and non-human species. In most non-human species, the need to identify subfertile animals is initiated from monetary concerns and is based on maximizing reproductive efficiency. This is particularly true in the case of the stallion. The routine clinical evaluation of stallion fertility has traditionally been based on the assessment of semen characteristics, including seminal volume, concentration of spermatozoa in the semen, percentage of motile spermatozoa in the semen and gross morphological assessment of the spermatozoa in the ejaculate (Dott, 1975).

Classification of normal sperm morphology has been shown to be important in the clinical evaluation of subfertility in stallions (Jasko et al., 1990) as well as a number of other species (Saake, 1972; Chandler et al., 1988). In bulls, an increase in the number of morphologically abnormal spermatozoa appears to be an indicator of seasonal variation of fertility (Sekoni and Gustafsson, 1987) and genetically heritable sperm anomalies (Hafez, 1987). A number of studies have indicated that evaluation of sperm morphology is an important component in the clinical assessment of human male fertility (Kruger et al., 1988;

Revised manuscript received 19 April 1996.
Chan et al., 1989) as well. The criteria for estimating the number of morphologically normal spermatozoa in the ejaculate of stallions has been well defined (Bielanski, 1951; Dott, 1975). Applying similar criteria, Jasko et al. (1990) showed that normal sperm morphology was highly correlated with fertility in studies using a large number of stallions. Normal sperm morphology has also been correlated with fertility in men (Kruger et al., 1988, Menkveld et al., 1990). However, the clinical evaluation of human sperm morphology includes criteria for normal metric dimensions of sperm heads (Kruger et al., 1988; WHO, 1992). The overall metric dimensions of the human sperm head (length/width) have been correlated with fertility (Katz et al, 1986). While the metric dimensions of sperm heads from clinically normal stallions have been previously reported (Bielanski, 1951), these dimensions have not been applied to the routine clinical evaluation of equine spermatozoa. Additionally, metric criteria of sperm heads has only recently been applied to studies of sperm morphology in fertile and subfertile stallions. Recent reports by Casey et al. (in press) indicate that morphometric differences occur in the measurements of sperm heads of fertile and subfertile stallions.

In defining the criteria for normal morphology of stallion spermatozoa, previous attempts by Bielanski (1951) to quantify the measurements of sperm heads were performed by manual, 
subjective methods. In studies of the assessment of human sperm morphology, similar subjective methods for evaluating the percentage of morphologically normal spermatozoa have been shown to be highly variable in nature (Baker and Clarke, 1987). In an attempt to reduce the subjectivity of the assessment of sperm head morphometry, a number of computer automated sperm morphometry analysis (ASMA) systems have been developed (Jagoe et al., 1986; Katz et al., 1986; Moruzzi et al., 1988). One such system has recently been used in the morphometric evaluation of stallion spermatozoa (Ball and Mohammed, 1995). While these one-of-a-kind systems provide valuable information and perform accurately within a research laboratory, they have not been commercially available for consistent evaluation in a number of laboratories. However, ASMA systems are now commercially available for the analysis of human (Davis et al., 1992; Kruger et al., 1993) and stallion spermatozoa (Davis et al., 1993). The operation of these commercial systems can be standardized (Davis and Gravance, 1993; Davis et al., 1993) and uniformly applied.

Commercial ASMA systems have been shown to be accurate and precise in measuring the dimensions of sperm heads of a number of species including stallions (Davis et al., 1993). Davis et al. (1993) have shown the importance of applying standard semen processing procedures as well as describing the optimum operational conditions for accurate ASMA analysis of the dimensions of stallion sperm heads. Applying ASMA to stallion sperm head morphometry, Casey et al. (in press) were able to determine that morphometric differences occurred between fertile and subfertile animals. While significant differences in morphometry were detected in mean group values, the criteria by which individual spermatozoa could be classified as 'normal' or 'abnormal' were not determined. In order to report the percentage of normal spermatozoa in a sample clinically, individual spermatozoa must be classified as normal or abnormal. In the clinical evaluation of human spermatozoa this goal is accomplished by application of a normal range of values for sperm head length, width and width/length. In the current study, the accuracy and precision of an ASMA instrument was used to begin to define the range of morphometric parameters of normal sperm heads from fertile stallions.

\section{Materials and Methods}

Semen samples were collected from 10 fertile and 10 subfertile stallions of various ages. The fertility of each stallion was determined by examination of extensive breeding records. Fertile stallions were defined as having greater than $60 \%$ conception rate per mare cycle. Stallions classified as subfertile had lower than $40 \%$ conception rate per cycle. Conception rates for all stallions were based on the same single breeding season. All semen samples were collected from stallions using a Missouri style AV. Immediately after collection, the semen was filtered, extended in skim milk extender (Casey et al., 1991) and maintained at room temperature until slides for morphometric analysis were prepared.

Slides were prepared by a standardized technique (Davis et al., 1993). A $200 \mu \mathrm{l}$ aliquot of semen from each sample was placed in a $1.5 \mu \mathrm{l}$ Eppendorf tube, diluted with $200 \mu \mathrm{l}$ Dulbecco's phosphate-buffered saline (DPBS), and then centrifuged at $600 \mathrm{~g}$ for $10 \mathrm{~min}$. The supernatant was removed and the pellet of spermatozoa resuspended in the correct volume of DPBS to achieve a final concentration of $200 \times 10^{6}$ cells ml $^{-1}$. Seven microlitres of the washed sperm sample was placed on the clear end of a frosted-end slide and dragged across the slide to create a fine feathered smear. Slides were allowed to dry in air for a minimum of $2 \mathrm{~h}$ before being stained for $40 \mathrm{~min}$ in Harris' haematoxylin (Davis et al., 1993). The morphometric dimensions of at least 200 properly digitized sperm heads were acquired for each stallion using an automated sperm head morphometry analysis system (Hamilton-Thorn Research, Beverly, MA) as previously described by Davis et al. (1993). Sperm heads were analysed at an objective magnification of $\times 40$ and photo-ocular magnification of $\times 6.7$. The metric measurements for the parameters of length, width, area, perimeter and width/length for each properly digitized sperm head of each analysis were saved in the computer for further statistical analysis. Moruzzi et al. (1988) found that ASMA could correctly classify human sperm head morphology with $95 \%$ accuracy applying these five parameters to human spermatozoa. The measurements for each sperm head analysed were then entered into a statistical database for further analysis ( $n>2000$ sperm per group). The mean metric measurements for area, perimeter, length, width and width/length of each stallion were recorded and the effects of group and stallion on sperm head dimensions were determined by general linear models analysis of variance (GLM-ANOVA, NCSS, Kaysville, UT). Group means were compared by Fischer's LSD test.

All sperm head measurements within each group (that is, from fertile or subfertile stallions) were then clustered by length, width, area, perimeter and width/length using iterative $k$-means cluster analysis techniques (NCSS). The number of clusters to create was determined when the explained variance between clustering steps was equal to or less than $5 \%$ (Davis et al., 1995a) for all parameters. The final number of clusters was set at five. The means, coefficients of variation (CV), $10 \%-90 \%$ range of values and number of sperm heads for each cluster were recorded for each stallion and group. In addition, the two major clusters of sperm head measures were combined to represent the major cluster (cluster FI) of fertile stallions. The effects of clusters within and between groups for the measurements of length, width, area, perimeter and width/ length were analysed by GLM-ANOVA. The mean measurements of sperm heads from the various clusters were compared by Fisher's LSD test.

The ranges of values from the 10th to the 90th percentile of length, width, area, perimeter and width/length from the FI major cluster of fertile stallions were then designated as 'normal' sperm head dimensions. These criteria for normal sperm head measurements (rounded to the nearest $0.1 \mu \mathrm{m}$ ) were then applied to all spermatozoa of all stallions to determine the percentage of spermatozoa with normal heads for each stallion. The percentage of normal sperm heads for each stallion was then arcsine transformed (Davis and Gravance, 1993). The effects of group and stallion on the percentage of normal sperm heads from the semen sample of each stallion were determined by GLM-ANOVA. The differences between groups were compared by Fisher's LSD test. 
Table 1. Mean morphometric values of all sperm heads from fertile and subfertile stallions

\begin{tabular}{lccccc} 
Group & $\begin{array}{c}\text { Length } \\
(\mu \mathrm{m})\end{array}$ & $\begin{array}{c}\text { Width } \\
(\mu \mathrm{m})\end{array}$ & $\begin{array}{c}\text { Area } \\
\left(\mu \mathrm{m}^{2}\right)\end{array}$ & $\begin{array}{c}\text { Perimeter } \\
(\mu \mathrm{m})\end{array}$ & $\begin{array}{c}\text { Width/ } \\
\text { length }\end{array}$ \\
\hline $\begin{array}{l}\text { Fertile stallions } \\
(n=2249)\end{array}$ & $5.35^{*}$ & $2.79^{*}$ & $11.43^{*}$ & $13.76^{*}$ & 0.52 \\
$\begin{array}{l}\text { Subfertile stallions } \\
(n=2260)\end{array}$ & $5.81^{*}$ & $2.90^{*}$ & $12.66^{*}$ & $14.68^{*}$ & 0.50 \\
\hline
\end{tabular}

*Values within columns are significantly different $(P<0.001)$

Table 2. Mean values and number of sperm heads in each generated cluster for length, width, area, perimeter and width/ length for sperm heads from fertile stallions

\begin{tabular}{|c|c|c|c|c|c|}
\hline \multirow[b]{2}{*}{ Parameter } & \multicolumn{5}{|c|}{ Cluster } \\
\hline & 1 & 2 & 3 & 4 & 5 \\
\hline Length $(\mu \mathrm{m})$ & 5.49 & 5.13 & 5.83 & 4.78 & 5.24 \\
\hline Width $(\mu \mathrm{m})$ & 2.65 & 2.85 & 2.88 & 2.47 & 3.38 \\
\hline Area $\left(\mu \mathrm{m}^{2}\right)$ & 11.22 & 11.24 & 12.74 & 9.08 & 13.23 \\
\hline Perimeter $(\mu \mathrm{m})$ & 13.79 & 13.40 & 14.73 & 12.30 & 14.59 \\
\hline Width/length & 0.49 & 0.55 & 0.49 & 0.52 & 0.65 \\
\hline$n$ & 606 & 559 & 529 & 365 & 190 \\
\hline
\end{tabular}

\section{Results}

A total of 2249 properly digitized spermatozoa from the fertile group and 2260 spermatozoa from the subfertile group were analysed. The mean morphometric measurements for length, width, area, perimeter and width/length of fertile and subfertile stallions are summarized (Table I). The values for all measures of sperm head dimensions were determined to be normally distributed by K.S. Normality Test (NCSS). Analysis of variance showed a significant effect of stallion $(P<0.001)$ and group $(P<0.001)$. The mean measurements for all sperm heads for all stallions within each group was significantly $(P<0.001)$ higher for length, width, area and perimeter in the subfertile group. Additionally, spermatozoa tended to be more tapered (lower width/length) in the subfertile group.

The percentage of sperm heads falling into the major cluster from normal stallions was 26.9 compared with 33.7 in the major cluster of sperm head dimensions of subfertile stallions (Tables $2,3)$. The variation within fertile stallions for the five measurement variables ranged from $3.6 \%$ for length to $6.2 \%$ for width/ length. The greatest number of spermatozoa fell into cluster 1 for five stallions while the largest membership for another three stallions was found in cluster 2 (Table 4 ). Because $80 \%$ of fertile stallions had the largest sperm membership in these two groups, the groups were combined to create a single cluster (FI) to represent the major cluster of sperm head dimensions of fertile stallions. The percentage of sperm head measurements for all fertile stallions falling into the F1 cluster was $51.8 \%$.

The mean values for length, width, area and perimeter of the major cluster of spermatozoa from the subfertile stallions were
Table 3. Mean values and number of sperm heads in each generated cluster for length, width, area, perimeter and width/ length for sperm heads from subfertile stallions

\begin{tabular}{lcrrrr}
\hline & \multicolumn{5}{c}{ Cluster } \\
\cline { 2 - 6 } Parameter & \multicolumn{1}{c}{1} & 2 & 3 & 4 & 5 \\
\hline & & & & & \\
Length $(\mu \mathrm{m})$ & 5.73 & 5.58 & 6.32 & 5.85 & 4.65 \\
Width $(\mu \mathrm{m})$ & 2.99 & 2.71 & 2.77 & 3.33 & 2.89 \\
Area $\left(\mu \mathrm{m}^{2}\right)$ & 12.95 & 11.45 & 13.05 & 14.54 & 9.99 \\
Perimeter $(\mu \mathrm{m})$ & 14.64 & 13.95 & 15.45 & 15.57 & 12.61 \\
Width/length & 0.52 & 0.49 & 0.45 & 0.57 & 0.63 \\
$n$ & 761 & 599 & 528 & 283 & 89 \\
& & & & & \\
\hline
\end{tabular}

Table 4. Number of sperm heads falling into each generated cluster for all fertile stallions

\begin{tabular}{|c|c|c|c|c|c|}
\hline \multirow{2}{*}{$\begin{array}{l}\text { Stallion } \\
\text { number }\end{array}$} & \multicolumn{5}{|c|}{ Cluster } \\
\hline & 1 & 2 & 3 & 4 & 5 \\
\hline 1 & 94 & 34 & 32 & 28 & 30 \\
\hline 2 & 29 & 38 & 11 & 140 & 5 \\
\hline 3 & 35 & 111 & 17 & 48 & 11 \\
\hline 4 & 29 & 107 & 15 & 49 & 23 \\
\hline 5 & 26 & 111 & 75 & 19 & 10 \\
\hline 6 & 87 & 35 & 80 & 13 & 13 \\
\hline 7 & 86 & 30 & 76 & 15 & 22 \\
\hline 8 & 34 & 63 & 113 & 8 & 9 \\
\hline 9 & 101 & 11 & 55 & 16 & 33 \\
\hline 10 & 85 & 19 & 55 & 29 & 34 \\
\hline Total & 606 & 559 & 529 & 365 & 190 \\
\hline
\end{tabular}

Table 5. Mean values of length, width, area, perimeter and width/length for the major clusters of spermatozoa from fertile and subfertile stallions

\begin{tabular}{lccccc}
\hline \multicolumn{5}{c}{ Parameter } \\
\cline { 2 - 6 } Group & $\begin{array}{c}\text { Area } \\
\left(\mu \mathrm{m}^{2}\right)\end{array}$ & $\begin{array}{c}\text { Perimeter } \\
(\mu \mathrm{m})\end{array}$ & $\begin{array}{c}\text { Length } \\
(\mu \mathrm{m})\end{array}$ & $\begin{array}{c}\text { Width } \\
(\mu \mathrm{m})\end{array}$ & $\begin{array}{c}\text { Width/ } \\
\text { length }\end{array}$ \\
\hline Fertile & $11.22^{*}$ & $13.79^{*}$ & $5.49^{*}$ & $2.65^{*}$ & $0.49^{*}$ \\
Subfertile & $12.95^{*}$ & $14.65^{*}$ & $5.73^{*}$ & $2.99^{*}$ & $0.52^{*}$ \\
\hline
\end{tabular}

*Values within columns are significantly different $(P<0.01)$.

significantly larger $(P<0.01)$ than those of the FI major cluster of the fertile group (Table 5). The $10 \%$ to $90 \%$ range of values for length, width, area, perimeter and width/length of spermatozoa falling into the two major clusters and the FI cluster of the fertile group and the major cluster of the subfertile group are summarized (Table 6).

The mean percentage (untransformed) of 'normal' sperm head morphometry for fertile and subfertile groups based on the ranges of values of the FI major cluster of the fertile 
Table 6. Range of values (10th-90th percentile) for length, width, area, perimeter and width/length and number of spermatozoa for the major clusters of spermatozoa from fertile and subfertile stallions

\begin{tabular}{lcccc}
\hline & \multicolumn{4}{c}{ Group } \\
\cline { 2 - 5 } Parameter & Fertile I & Fertile 2 & Fertile & Subfertile I \\
\hline Area $\left(\mu \mathrm{m}^{2}\right)$ & $10.3-11.9$ & $10.4-12.1$ & $10.3-12.1$ & $12.2-13.7$ \\
Perimeter $(\mu \mathrm{m})$ & $13.3-14.2$ & $12.9-14.0$ & $12.9-14.2$ & $14.1-15.1$ \\
Length $(\mu \mathrm{m})$ & $5.3-5.7$ & $4.9-5.4$ & $4.9-5.7$ & $5.4-6.0$ \\
Width $(\mu \mathrm{m})$ & $2.5-2.8$ & $2.7-3.0$ & $2.5-3.0$ & $2.9-3.1$ \\
Width/length & $0.45-0.51$ & $0.52-0.59$ & $0.45-0.59$ & $0.49-0.57$ \\
$n$ & 606 & 559 & 1165 & 761 \\
\hline
\end{tabular}

Fertile 1: major cluster of sperm head dimensions of fertile stallions (cluster 1). Fertile 2: second most populated cluster of sperm head dimensions of fertile stallions (cluster 2).

Fertile: range of values for two major clusters of sperm head dimensions of fertile stallions.

Subfertile 1: major cluster of sperm head dimensions of subfertile stallions (cluster 1).

Table 7. Mean percentage of normal sperm head measurements and coefficient of variation (CV) for fertile and subfertile stallions

\begin{tabular}{llc} 
& \multicolumn{2}{c}{ Percentage normal } \\
\cline { 2 - 3 } $\begin{array}{l}\text { Stallion } \\
\text { number }\end{array}$ & Fertile & Subfertile \\
\hline
\end{tabular}

\begin{tabular}{llc}
\hline 1 & 58 & 41 \\
2 & 30 & 23 \\
3 & 66 & 30 \\
4 & 61 & 6 \\
5 & 57 & 26 \\
6 & 54 & 9.5 \\
7 & 51 & 11 \\
8 & 43 & 14 \\
9 & 52 & 20 \\
10 & 46 & 5.1 \\
Mean & $52^{*}$ & $19^{*}$ \\
CV & 20 & 62 \\
\hline
\end{tabular}

${ }^{*}$ Mean values are significantly different $(P<0.001)$.

stallions in Table 6 are given (Table 7). The mean percentage of normal sperm head morphometry was significantly higher $(P<0.001)$ in the fertile group compared with the subfertile group. The percentage of normal sperm head morphometry ranged from $30 \%$ to $66 \%$ among fertile stallions and from $5.1 \%$ to $41 \%$ in the subfertile stallions. The variation for the mean percentage of normal sperm head morphometry within groups was much higher in the subfertile group as well.

\section{Discussion}

In the present study, over 2000 spermatozoa representing 10 fertile and 10 subfertile stallions were analysed in an attempt to quantify the morphometric dimensions of sperm heads from stallions. As observed previously (Casey et al., in press), the group mean morphometric dimensions were significantly larger for area, perimeter and length in subfertile stallions. In addition, the width was found to be significantly greater for subfertile stallions in the present study, when the means of all sperm heads in each group were compared. The determination of significant differences in the morphometric dimensions, even when significant differences are present, is probably due to the accurate and objective measurements obtained by the ASMA system.

While determining that morphometric differences occur between two gross populations is biologically notable, it can be difficult to apply morphometric measurements to the clinical assessment of fertility. In performing a typical routine semen evaluation, the morphological assessment of spermatozoa is determined and reported as the percentage of normal spermatozoa in the sample. In the determination of morphologically normal spermatozoa in a human ejaculate, morphometric criteria for a normal sperm head have been defined (Kruger et al., 1988; Menkveld et al., 1991; WHO, 1992). While the general size and shape of stallion sperm heads have been described (Bielanski, 1951), these observations were performed using manual and subjective methods. Furthermore, these measurements have not been applied as a component of clinical evaluation of fertility. In the clinical evaluation of stallion sperm morphology, it appears that application of such criteria may add significant information and play an important role in the detection of subfertility.

In determining the shape and size of normal human sperm heads, the criteria have been based on selecting aesthetically pleasing forms of spermatozoa. In recent years, meticulous observation of sperm head dimensions and application of determined measurements to biological outcome (Katz et al., 1986; Kruger et al., 1988) have led to changes in the definition of normal sperm head morphometry (WHO, 1987, 1992; Kruger et al., 1988; Menkveld et al., 1990; Menkveld et al., 1991; Davis and Gravance, 1994). The application of these numerous criteria to the determination of percentage of normal spermatozoa in a sample, when observed by a large number of individuals, is widely variable (Davis et al., 1995b). In order to avoid similar confusion in applying metric criteria to 'normal' head dimensions of stallion spermatozoa, the current study combined accurate and precise objective ASMA analysis with powerful multivariate statistical cluster analysis to determine objectively the major subpopulations of spermatozoa within fertile stallions. These measurements were presumed to be of the normal population, since they were obtained from the major population of fertile males.

When multivariate analysis was applied to the groups of sperm head dimensions, discrete subpopulations (clusters) were generated based on the set number of five clusters. In the analysis of subpopulations of sperm head dimensions, significant differences in the values for length, width, area and perimeter were found between the major subpopulations of sperm heads of fertile and subfertile stallions. The differences in cluster populations were analysed across all sperm heads and stallions within groups, rather than across stallions, using mean values of major clusters of individual stallions. Analysing all sperm heads of fertile stallions as a group allows for the 
detection of natural variation of the individual populations between fertile stallions. For example, if the range of values of sperm head measurements of the major cluster from stallion 2 in the fertile group was combined with those of major clusters of the remaining stallions, the percentage of sperm measurements in the cluster would have been falsely increased to a value greater than $30 \%$. In addition, this type of approach would have increased the range of the major cluster downward for the remaining stallions to a range of values where only a small percentage of their sperm head dimensions actually existed.

Since eight of the ten fertile stallions were found to have the greatest number of sperm heads whose dimensions fell in cluster 1 or cluster 2 , the means and ranges of the two groups were combined for defining the dimensions of sperm heads of fertile stallions. Combining the two clusters yielded a majority $(51.8 \%)$ of sperm heads falling into the major cluster and yielded a net range of values for width, length and width/length similar to that of human spermatozoa (WHO, 1992). In order to further remove any outlying values, the final criteria for morphometric measurements of normal stallion sperm heads were selected as the 10th-90th percentile of values within each parameter for all spermatozoa within the group of spermatozoa from fertile stallions. The majority of spermatozoa from the semen of fertile stallions was assumed to be normal; thus the range of values from the major cluster was considered to have the 'normal' dimensions for stallion sperm heads.

The percentage of normal sperm heads for each stallion was then determined by applying the metric criteria of normal sperm head morphometry to all spermatozoa from all stallions. The percentage of sperm heads with normal morphometry, based on various criteria, is generated when human spermatozoa are analysed by a similar ASMA system (Cell-Form Human ${ }^{(}{ }^{3}$, Motion Analysis Corp, Santa Rosa, CA). Additionally, this type of report is consistent with, and may be applied to, traditional clinical reports of sperm morphology assessment.

The percentage of sperm heads with normal morphometry in fertile stallions ranged from $30 \%$ to $66 \%$, whereas values for subfertile stallions ranged from $5.1 \%$ to $40 \%$. Only one stallion in the subfertile group had more than $30 \%$ of spermatozoa with normal heads, while one stallion in the fertile group had less than $40 \%$ normal sperm head morphometry. These results suggest that a value of $<30 \%$ of spermatozoa with normal head morphometry may indicate impaired fertility in stallions, while a value $>40 \%$ would be indicative of a fertile stallion. The range of $30 \%-40 \%$ of spermatozoa with normal head morphometry appears to be the threshold level of impaired fertility. By applying the powerful techniques of ASMA and multivariate cluster analysis, it was possible to determine discrete subpopulations of sperm head dimensions in fertile and subfertile stallions accurately. Normal values were then defined using the morphometric values of the major populations of sperm head dimensions of normal stallions. Significant differences in the percentage of normal sperm heads were then used to distinguish between fertile and subfertile stallions by applying these ranges of values to individual spermatozoa. It is thus possible to apply objective ASMA analysis of sperm head morphometry to clinically relevant endpoints.

This project was supported by the California Center for Equine Health and Performance (formerly The Equine Research Laboratory),
University of California, Davis, CA, with funds provided by the Oak Tree Racing Association, the State of California Satellite Wagering Fund, and contributions from private donors. The authors would also like to thank Hamilton Thorn Research (Beverly MA) for providing technical assistance.

\section{References}

Baker HWG and Clarke GN (1987) Sperm morphology: consistency of assessment of the same sperm by different observers Clinical Reproduction and Fertility $5 \quad 37-43$

Ball BA and Mohammed HO (1995) Morphometry of stallion spermatozoa by computer assisted image analysis Theriogenology 44 367-377

Bielanski $W$ (1951) Characteristics of the semen of stallions: macro and microscopic investigations with estimation of fertility Mem Academy of Polish Science, Letter B16

Casey PJ, Liu IKM, Stewart DR and Scott MA (1991) The effects of equine relaxin on sperm longevity and motility in chilled and cryopreserved semen Journal of Reproduction and Fertility Supplement 44 645-646

Casey PJ, Gravance CG, Davis RO, Chabot DD and Liu IKM Morphometric differences in sperm head dimensions of fertile and subfertile stallions Theriogenology (in press)

Chan SYW, Wang C, Chan STH, Ho PC, So WWK, Chan YF and Ma HK (1989) Predictive value of sperm morphology and movement characteristics in the outcome of in vitro fertilization of human oocytes journal of In Vitro Fertilization and Embryo Transfer 6 142-148

Chandler JE, Painter CL, Adkinson RW, Memon MA and Hoyt PG (1988) Semen quality characteristics of dairy goats Journal of Dairy Science 71 1638-1646

Davis RO and Gravance CG (1994) Consistency of sperm morphology classification methods Journal of Andrology 15 83-91

Davis RO, Bain DE, Siemers RJ, Thal DM, Andrew JB and Gravance CG (1992) Accuracy and precision of the CellForm-Human automated sperm morphometry system Fertility and Sterility 58 763-769

Davis RO, Gravance CG and Casey PJ (1993) Automated morphometric analysis of stallion spermatozoa American Journal of Veterinary Research 54 18081811

Davis RO, Drobnis EZ and Overstreet JW (1995a) Application of multivariate cluster, discriminate function, and stepwise regression analyses to variable selection and predictive modeling of sperm cryosurvival Fertility and Sterility $631051-1057$

Davis RO, Gravance CG and Overstreet JW (1995b) A standardized test for the visual analysis of human sperm morphology Fertility and Sterility 63 1058-1063

Dott HM (1975) Morphology of stallion spermatozoa Joumal of Reproduction and Fertility Supplement 23 41-46

Hafez ESE (1987) Semen Evaluation. In Reproduction in Farm Animals, 5th Edn pp. 455-480 Ed. ESE Hafez. Lea and Febiger, Philadelphia

Jagoe JP, Washbrook NP and Hudson EA (1986) Morphometry of spermatozoa using semiautomatic image analysis Journal of Clinical Pathology 39 13471352

Jasko DJ, Lein DH and Foote RH (1990) Determination of the relationship between sperm morphologic classification and fertility in stallion: 66 cases (1987-1988) Journal of the American Veterinary Medical Association 197 $389-394$

Katz DF, Overstreet JW, Samuels SJ, Niswander PW, Bloom TD and Lewis EL (1986) Morphometric analysis of spermatozoa in the assessment of human male fertility Journal of Andrology 7 203-210

Kruger TF, Acosta AA, Simmons KF, Swanson RJ, Matta JF and Oehninger S (1988) Predictive value of abnormal sperm morphology in in vitro fertilization Fertility and Sterility 49 112-117

Kruger TF, Tuitot TC, Franken DR, Acosta AA, Oehninger SC, Menkveld R and Lombard CJ (1993) A new computerized method of reading sperm morphology (strict criteria) is as efficient as technician reading Fertility and Sterility 59 202-209

Menkveld R, Stander FSH, Kotze TJ, Kruger TF and van Zyle JA (1990) The evaluation of morphological characteristics of human spermatozoa according to stricter criteria Human Reproduction 5 586-592

Menkveld R, Oettle EE, Kruger TF, Swanson RJ, Acosta AA and Oehninger S (1991) Atlas of Human Sperm Morphology, 1st Edn. Williams and Wilkins, Baltimore, MD 
Moruzzi JF, Wyrobek AJ, Mayall BH and Gledhill BL (1988) Quantification and classification of human sperm morphology by computer-assisted image analysis Fertility and Sterility 50 142-152

Saake RG (1972) Semen quality test and their relationship to fertility $N A A B$ Proceedings of the 4th Technical Conference on Animal Reproduction and Fertility, pp 22-28

Sekoni VO and Gustafsson BK (1987) Seasonal variations in the incidence of sperm morphological abnormalities in dairy bulls regularly used for artificial insemination British Veterinary Journal 143 312-317
World Health Organisation (1987) WHO Laboratory Manual for the Examination of Human Semen and Sperm-Cervical Mucus Interaction, 2nd Edn. Cambridge University Press, Cambridge

World Health Organisation (1992) WHO Laboratory Manual for the Examination of Human Semen and Semen-Cervical Mucus Interaction, 3rd Edn. Cambridge University Press, Cambridge 